\title{
Comparison of surgical techniques for early-stage thymoma: Feasibility of minimally invasive thymectomy and comparison with open resection
}

\author{
Arjun Pennathur, MD, ${ }^{\mathrm{a}}$ Irfan Qureshi, MD, ${ }^{\mathrm{a}}$ Matthew J. Schuchert, MD, ${ }^{\mathrm{a}}$ Rajeev Dhupar, MD, ${ }^{\mathrm{b}}$ \\ Peter F. Ferson, MD, ${ }^{a}$ William E. Gooding, MS, ${ }^{\mathrm{c}}$ Neil A. Christie, MD, ${ }^{a}$ Sebastien Gilbert, MD, ${ }^{\mathrm{a}}$ \\ Manisha Shende, MD, ${ }^{\mathrm{a}}$ Omar Awais, DO, ${ }^{\mathrm{a}}$ Joel S. Greenberger, MD, ${ }^{\mathrm{d}}$ Rodney J. Landreneau, MD, ${ }^{\mathrm{a}}$ and \\ James D. Luketich, MD ${ }^{\mathrm{a}}$
}

\begin{abstract}
Objective: The minimally invasive, video-assisted thoracoscopic surgical (VATS) approach to resection of the thymus is frequently practiced for benign disease; however, a VATS approach for thymoma remains controversial. The objective of the present study was to evaluate the feasibility of VATS thymectomy for the treatment of early-stage thymoma and to compare the outcomes with those after open resection.
\end{abstract}

\begin{abstract}
Methods: A retrospective review of 40 patients who underwent surgical resection of early-stage thymoma during a 12-year period was conducted. Data on patient characteristics, morbidity, recurrence, and survival were collected. The primary endpoint studied was overall survival.
\end{abstract}

\begin{abstract}
Results: Of the 40 patients, 14 underwent thymectomy for stage I and 26 for stage II thymoma; 19 were men and 21 were women (median age, 64 years; range, 35-86 years). Open thymectomy was performed in 22 patients, and VATS was performed in 18 . The operative mortality rate was $0 \%$. The tumor stage and number of patients undergoing adjuvant radiotherapy were comparable in both surgical groups. The median length of hospital stay was shorter in the VATS group ( 3 days) than in the open group (5 days) $(P=.0001)$. The median follow-up was 36 months. No significant differences were found in the estimated recurrence-free and overall 5-year survival rates $(83 \%-100 \%)$ between the 2 groups.
\end{abstract}

Conclusions: VATS of early-stage thymoma appears safe and feasible and was associated with a shorter hospital stay. The oncologic outcomes were comparable in the open and VATS groups during intermediate-term follow-up. Additional follow-up is required to evaluate the long-term results of thoracoscopic thymectomy for early-stage thymoma. (J Thorac Cardiovasc Surg 2011;141:694-701)

Tumors of the mediastinum are relatively rare. ${ }^{1}$ Thymoma is the most common primary neoplasm of the mediastinum in the adult population, with surgery the primary treatment modality, except for very advanced stages. ${ }^{2}$ In approximately $30 \%$ of patients with thymoma, myasthenia gravis (MG) is coexistent. ${ }^{3}$ Several controversies exist with regard to thymomas, including the staging systems used, the approach to surgery, and the use of adjuvant therapy.

During the past 1 to 2 decades, minimally invasive approaches have been described for the performance of sev-

From the Heart, Lung, and Esophageal Surgery Institute, ${ }^{a}$ University of Pittsburgh Medical Center; the Department of Surgery, ${ }^{\mathrm{b}}$ University of Pittsburgh; and the University of Pittsburgh Cancer Institute Biostatistics Facility ${ }^{\mathrm{c}}$ and the Department of Radiation Oncology, ${ }^{\mathrm{d}}$ University of Pittsburgh Medical Center, Pittsburgh, Pa. Disclosures: Authors have nothing to disclose with regard to commercial support.

Read at the 90th Annual Meeting of The American Association for Thoracic Surgery, Toronto, Ontario, Canada, May 1-5, 2010.

Received for publication May 20, 2009; revisions received Sept 3, 2010; accepted for publication Sept 9, 2010; available ahead of print Jan 21, 2011.

Address for reprints: James D. Luketich, MD, Department of Cardiothoracic Surgery, University of Pittsburgh Medical Center, 200 Lothrop Street, C-800, Pittsburgh, PA 15213 (E-mail: luketichjd@upmc.edu).

$0022-5223 / \$ 36.00$

Copyright $₫ 2011$ Published by Elsevier Inc. on behalf of The American Association for Thoracic Surgery

doi:10.1016/j.jtcvs.2010.09.003 eral thoracic procedures. These have included treatment of benign diseases and the treatment of esophageal and lung cancer. ${ }^{4-8}$ Blalock and colleagues ${ }^{9}$ described a series of patients with MG with improvement after thymectomy. Since then, thymectomy has been an important component in the management of MG. ${ }^{9}$ In patients with thymoma, surgical resection with total thymectomy, performed using an open technique, typically with a sternotomy, has been the standard treatment. A recent systematic review of surgical resection for thymoma reported that the 5-year overall survival rate of patients with stage I and stage II thymoma ranged from $89 \%$ to $100 \%$ and $71 \%$ to $95 \%$, respectively. ${ }^{10}$ That review also concluded that the published evidence comparing various approaches, in particular, minimally invasive approaches, was insufficient with regard to the outcomes. Although some published data are available for thoracoscopic thymectomy for nonthymoma MG, very few data are available describing the outcomes after minimally invasive resection of thymoma.

The minimally invasive approaches described for the resection of thymus have included transcervical thymectomy and video-assisted thoracoscopic thymectomy. ${ }^{11-14}$ Although these minimally invasive thoracoscopic approaches to 


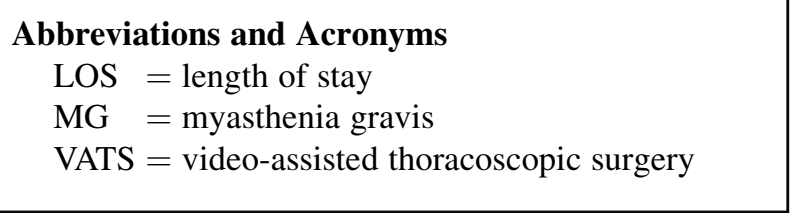

resection of the thymus are frequently practiced for benign disease, a video-assisted thoracoscopic surgical (VATS) approach for thymoma remains controversial. The primary objective of the present study was to evaluate the feasibility of VATS thymectomy for early-stage (stage I and II) thymoma. Our second objective was to compare the intermediate-term outcomes of a minimally invasive approach (VATS) with those after open resection.

\section{MATERIALS AND METHODS}

We retrospectively reviewed our experience with thymectomy for the treatment of early-stage (stage I and II) thymoma at the University of Pittsburgh (during a 12-year period 1996-2008). The institutional review board at the University of Pittsburgh approved the study.

\section{Patient Characteristics}

A total of 40 patients who underwent thymectomy for stage I or II thymoma were identified. Of the 40 patients, 14 had stage I thymoma and 26 had stage II thymoma; 19 were men and 21 were women (median age, 64 years; range, 35-86 years). Open thymectomy was performed in 22 patients, and VATS resection in 18. MG was present in 11 patients $(27.5 \%)$. The patient characteristics are summarized in Table 1 .

\section{Surgical Technique}

The technique of VATS thymectomy has been previously described ${ }^{11-13}$ and can be performed with a unilateral or bilateral approach. In brief, general endotracheal anesthesia was established with a double-lumen endotracheal tube or a single-lumen tube with carbon dioxide insufflation. For the unilateral right-sided approach, the ports were placed along the right anterior axillary line, conforming to the mammary crease. Three 5-mm ports were placed in the third, fifth, and eighth interspaces. After right lung isolation, the inferior portion of the thymus was mobilized. Dissection was started in the normal thymus, away from the tumor, with minimal manipulation of the tumor to avoid any breaches in the capsule. It is critical that the tumor manipulation is kept to a minimum and that the plane of dissection is kept beyond the capsule. Similarly, direct traction by grasping the tumor should be avoided to limit capsular tears and contamination locally and in the pleural space. The dissection plane was extended cranially, taking care to preserve the integrity of the right phrenic nerve. The left portion of the thymus was mobilized free from the pericardium overlying the ascending aorta. The thymic veins were clipped and divided. The right and left thymic horns were dissected free, and the specimen was removed in a plastic retrieval bag.

Our current approach has been bilateral VATS, which promotes better visualization of key anatomic structures and facilitates the accomplishment of complete thymectomy. We have typically used a single-lumen tube with carbon dioxide insufflation, with the patient in the supine position. The procedure was begun on the left, and a similar 5-mm port arrangement was established. The mediastinal pleura was incised just anterior to the phrenic nerve. The base of the left thymic lobe was mobilized and retracted superiorly. All thymic and perithymic fatty tissue was mobilized en bloc with the specimen, allowing exposure of the underlying pericardium and
TABLE 1. Patient characteristics

\begin{tabular}{lr}
\hline \multicolumn{1}{c}{ Characteristic } & Value \\
\hline Gender (n) & \\
Male & 19 \\
Female & 21 \\
Age (y) & \\
$\quad$ Median & 63 \\
Range & $35-86$ \\
Stage & \\
Stage I & 14 \\
Stage II & 26 \\
WHO histologic type & \\
A & 6 \\
AB & 12 \\
B1-B3 & 20 \\
C & 1 \\
Not determined & 1 \\
Surgical approach & \\
VATS & 18 \\
Open & 22
\end{tabular}

Stage and surgical approach

Stage 1

VATS

Open

Stage 2

VATS

Open

Median tumor size (cm)

VATS

$3.6^{*}$

Open

6.1

Myasthenia gravis

VATS

7

Open

4

VATS, Video-assisted thoracoscopic surgical resection; $W H O$, World Health Organization. $* P=.0003$.

aorta. The thymus was then carefully dissected along the innominate vein, and the thymic tributaries were clipped and divided. The dissection was continued to the innominate-superior vena cava junction. The cervical horns were mobilized and swept inferiorly with the specimen. Once maximal mobilization was achieved on the left, the ports and instrumentation were withdrawn. The lung was re-expanded with a red-rubber catheter positioned within the pleural space to evacuate the pneumothorax, and the catheter was removed. Similar to the left side, 3 ports were placed on the right side, and dissection was completed along the innominate-superior vena cava junction, the venous thymic branches were clipped, any residual attachments were divided, and the specimen was removed. The techniques for open total thymectomy have been well described previously. ${ }^{15}$

\section{Data Collection}

The objective of the present study was to determine the feasibility of a minimally invasive VATS approach for resection of early-stage thymoma and to compare the outcomes of minimally invasive VATS resection with those of standard open surgical resection. Information on patient demographics, co-morbidities, Charlson co-morbidity index, ${ }^{16}$ the presence of associated MG, tumor characteristics, staging information, surgical approach, and adjuvant treatment was collected. The Masaoka staging system was used to define the stage I and stage II tumors. ${ }^{17}$ In patients with MG, the Osserman score and the Myasthenia Gravis Foundation of America scores were collected. ${ }^{18,19}$ 


\section{Statistical Analysis}

The specific endpoints studied were perioperative complications, recurrence-free survival, and overall survival. The primary endpoint studied was overall survival, and the secondary endpoint was recurrence-free survival. Kaplan-Meier plots with $95 \%$ Greenwood confidence intervals were constructed to estimate the overall survival and recurrence-free survival. Patients who were alive at follow-up were censored from the analysis of overall survival. Recurrence-free survival was computed as the interval from resection to disease recurrence. For the present analysis, the patients who were alive at the last follow-up examination or who had died while disease free were censored. The patient characteristics in the open resection and VATS resection groups were compared using Fisher's exact test for categorical variables and the Wilcoxon test for continuous variables. The postoperative Osserman score was compared with the preoperative score using the signed rank test. All tests were 2 tailed.

A 2-way factorial analysis of variance was conducted to test the interaction between the surgeon and operative procedure. The analysis was limited to surgeons who had performed more than 4 procedures. The association between the Charlson co-morbidity index, and length of stay (LOS) was evaluated by testing the significance of the Spearman rank correlation coefficient.

\section{Propensity Score}

We compared the 2 surgical procedure groups by constructing a propensity score. ${ }^{20}$ The propensity score is a predicted score that is proportional to the likelihood that, according to the covariates, a patient has been chosen for either VATS or open surgery. The score has the tendency to equilibrate the comparison arms in an observational study as if the treatments had been randomly assigned. We evaluated the following candidates for their utility in a propensity score: Charlson co-morbidity index (raw and age adjusted), patient age and gender, tumor size and stage, date of surgery, and the presence of MG. We selected 3 covariates because of their association with the surgical procedure group: tumor size, date of surgery, and the presence of MG. For the evaluation of overall and recurrence-free survival, we used Cox proportional hazards regression analysis to test the effect of the procedure type, adjusting for the propensity score. To evaluate the effect on the LOS, we applied analysis of covariance, treating the propensity score as a continuous covariate.

\section{RESULTS}

\section{Comparison of Patient Characteristics}

In the present patient cohort, 22 patients underwent open thymectomy and 18 underwent VATS resection. The tumor stages were comparable in the 2 surgical groups, and no significant differences were found in the number of patients with MG or the number receiving adjuvant therapy between the 2 groups. The median tumor size in the VATS group was significantly smaller than that in the open group, with a median tumor size of $3.6 \mathrm{~cm}$ in the VATS group and $6.1 \mathrm{~cm}$ in the open group $(P=.0003)$. These analyses are summarized in Table 2.

\section{Comparison of Outcomes}

The operative mortality rate was $0 \%$. The median hospital LOS was shorter for the VATS group (3 days) than for the open group (5 days) $(P=.0001)$ (Table 2$)$. Significant morbidity was infrequent, and the most frequent perioperative complications were atrial fibrillation $(\mathrm{n}=2)$, pneumonia
TABLE 2. Comparison of variables of VATS versus open surgical groups

\begin{tabular}{|c|c|c|c|}
\hline \multirow[b]{2}{*}{ Variable } & \multicolumn{2}{|c|}{ Surgery type } & \multirow[b]{2}{*}{$P$ value } \\
\hline & VATS & Open & \\
\hline Gender & & & $.525^{*}$ \\
\hline Male & 10 & 9 & \\
\hline Female & 8 & 13 & \\
\hline Median age (y) & 64 & 64 & $.924 \dagger$ \\
\hline CCI & 3 & 2 & $.294 \dagger$ \\
\hline Tumor stage & & & $.510 *$ \\
\hline I & 5 & 9 & \\
\hline II & 13 & 13 & \\
\hline Adjuvant therapy & & & $.360^{*}$ \\
\hline Yes & 7 & 12 & \\
\hline No & 11 & 10 & \\
\hline Margin status & & & $.196^{*}$ \\
\hline Positive & 1 & 0 & \\
\hline Negative & 16 & 22 & \\
\hline MG & & & $.1734 *$ \\
\hline Yes & 7 & 4 & \\
\hline No & 11 & 18 & \\
\hline Tumor size $(\mathrm{cm})$ & & & $.0003 \dagger$ \\
\hline Median & 3.6 & 6.1 & \\
\hline Mean & 3.5 & 5.8 & \\
\hline $\mathrm{SD}$ & 1.1 & 2 & \\
\hline $\operatorname{LOS}(\mathrm{d})$ & & & $.0001 \dagger$ \\
\hline Median & 3 & 5 & \\
\hline Mean & 2.9 & 6.2 & \\
\hline SD & 1.6 & 4.2 & \\
\hline
\end{tabular}

VATS, Video-assisted thoracoscopic surgical resection; $C C I$, Charlson co-morbidity index; $M G$, myasthenia gravis; $\mathrm{SD}$, standard deviation; $L O S$, length of stay. *Fisher's exact test. †Wilcoxon test (2 tailed).

$(\mathrm{n}=1)$, and respiratory insufficiency requiring ventilator support $(\mathrm{n}=2)$.

Of the 40 patients, 11 had MG, 7 in the VATS group and 4 in the open group. The MG symptoms, as assessed by the Myasthenia Gravis Foundation of America scale, improved in 9 of the 11 patients, were unchanged in 1 , and were exacerbated in 1 . The median modified Osserman score was 2 preoperatively. The median within-patient Osserman score had declined by 2 points from the preoperative score at the last follow-up assessment. This decrease (improvement) in the Osserman score was statistically significant (signed rank test, $P=.006$ ). No differences between the 2 surgical approaches and the improvement in Osserman score were noted.

\section{Overall Survival}

No procedure-related mortalities occurred. During the follow-up period, 3 patients died. The median follow-up for the remaining patients was 36 months (58 months for the open group and 27 months for the VATS group). The minimally invasive technique was introduced later in our series, resulting in the shorter median follow-up for the VATS group. The estimated probability of 5-year overall survival 


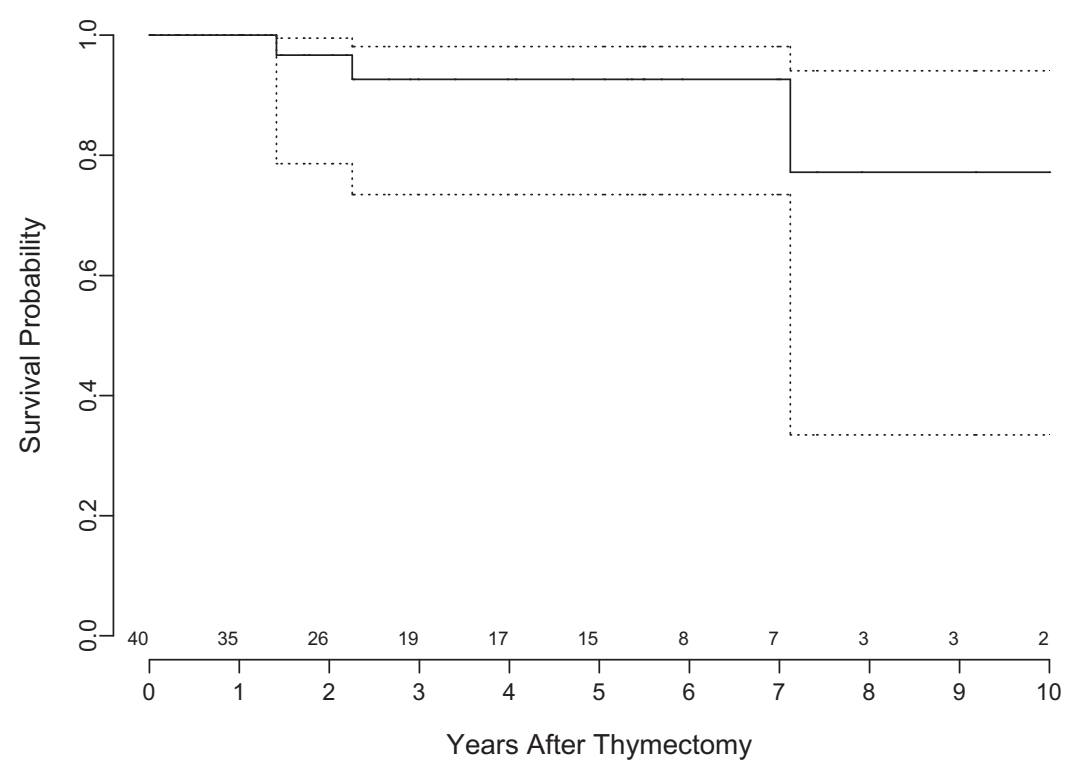

FIGURE 1. Kaplan-Meier plot illustrating overall survival for entire group, with confidence limits. Time, in years, from thymectomy plotted on $x$-axis. Dotted lines indicate $95 \%$ confidence bands for probability of overall survival. Numbers at risk shown above x-axis.

for the entire cohort was $93 \%$ (95\% confidence interval, $74 \%-98 \%$ ) (Figure 1). When the surgical approaches were compared, the probability of estimated 5-year overall survival was $100 \%$ for the VATS group and $88 \%$ ( $95 \%$ confidence interval 59-97\%) for the open group $(P=.949)$. There was no significant difference in overall survival when the surgical approach was stratified by tumor stage.

\section{Recurrence-Free Survival}

During follow-up, 1 patient developed a recurrence. The estimated probability of 5-year recurrence-free survival for the entire cohort was $93 \%$ (95\% confidence interval, $59 \%-99 \%$ ) (Figure 2). When the surgical approaches were compared, the estimated 5-year recurrence-free survival rate was $100 \%$ for the VATS group and $83 \%$ ( $95 \%$ confidence interval, $27 \%-88 \%$ ) for the open group $(P=.602)$ (Figure 3, $A)$. There were no significant differences in recurrence-free survival when the surgical approach was stratified by tumor stage. Figure $3, B$ and $C$, depicts the results of estimated recurrence-free survival of patients with stage I and stage II thymoma, stratified by the surgical approach.

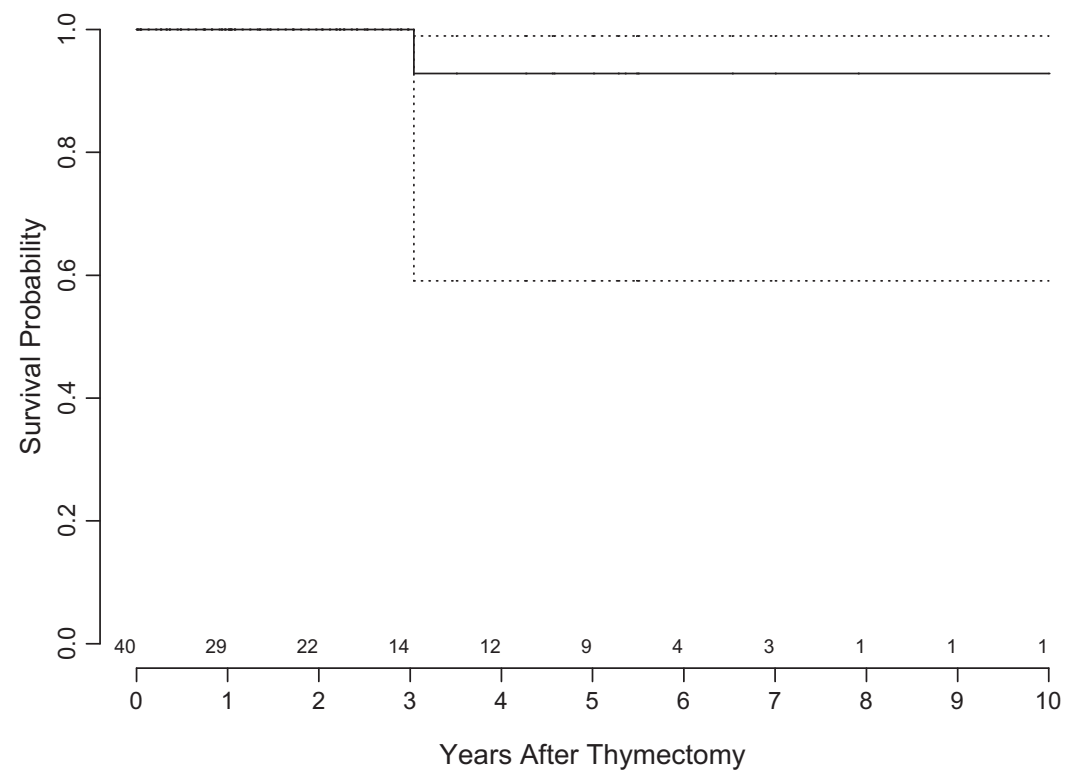

FIGURE 2. Kaplan-Meier plot illustrating recurrence-free survival for entire group, with confidence limits. Time, in years, from thymectomy plotted on $\mathrm{x}$-axis. Dotted lines indicate $95 \%$ confidence bands for probability of recurrence-free survival. Numbers at risk shown above $\mathrm{x}$-axis. 

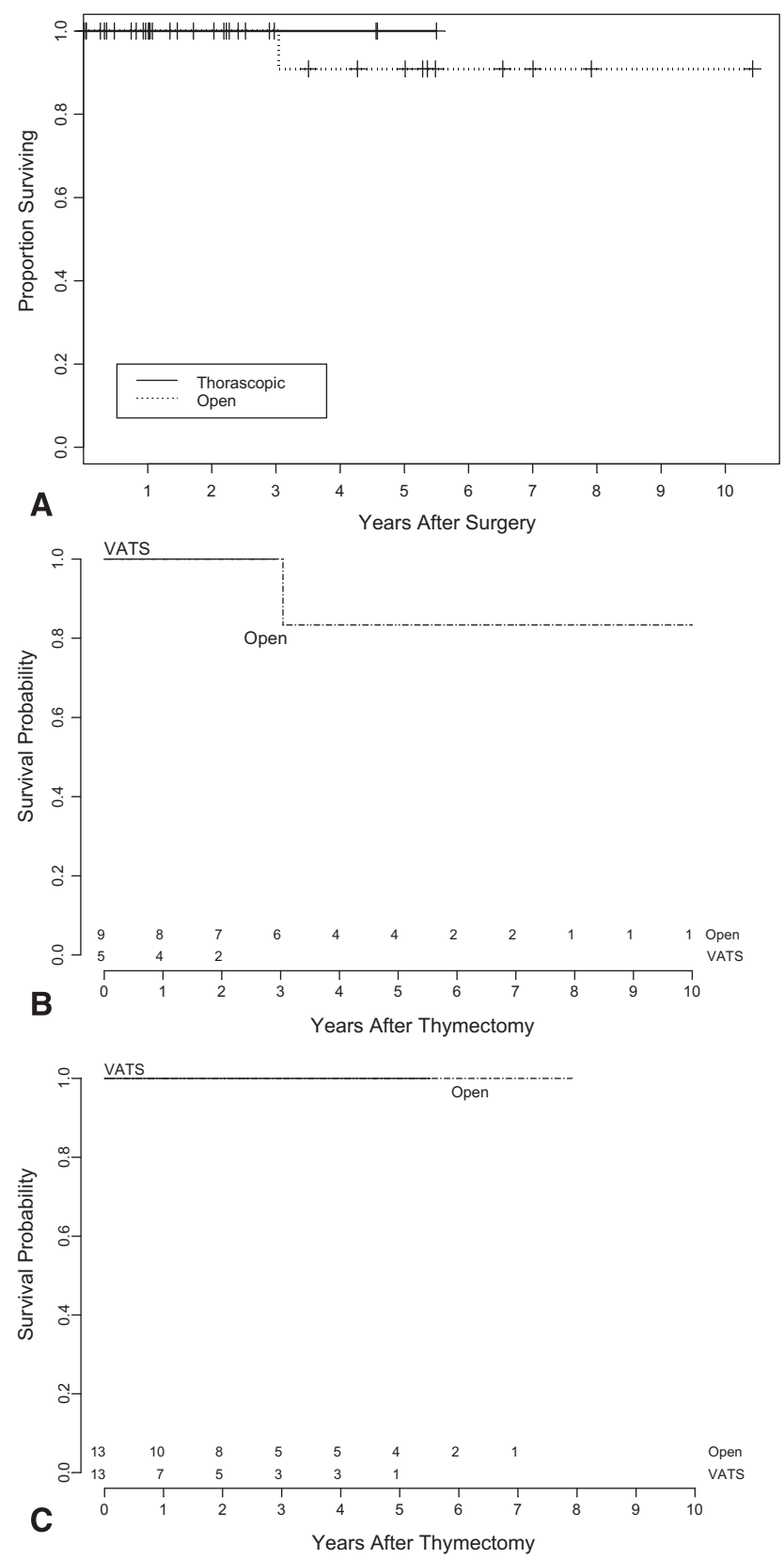

FIGURE 3. A, Kaplan-Meier plot illustrating recurrence-free survival stratified by group (thoracoscopic vs open). Time, in years, from thymectomy plotted on X-axis. B, Kaplan-Meier plot illustrating recurrence-free survival for patients with stage I thymoma stratified by group (thoracoscopic vs open). Time, in years, from thymectomy plotted on x-axis. Numbers at risk shown above x-axis. C, Kaplan-Meier plot illustrating recurrence-free survival for patients with stage II thymoma stratified by group (thoracoscopic vs open). Time, in years, from thymectomy plotted on $\mathrm{x}$-axis. Numbers at risk shown above $\mathrm{x}$-axis.

\section{Surgeon and LOS}

Five surgeons performed most of the operations, and each performed 4 or more procedures. Of the 5 surgeons, 4 performed both VATS and open resection, and 1 surgeon exclu- sively performed open resection. The median LOS of patients treated by the busier surgeons showed that an open approach was associated with a longer LOS. A 2way factorial analysis of variance was conducted to test the interaction between surgeon and operative procedure among the surgeons who performed most of the procedures.

This test of interaction indicated that no interaction was present between the surgeon and procedure type (ie, the effect of the procedure was independent of the surgeon performing the procedure). Among the 4 busy surgeons who did both procedures, the LOS was consistently longer for those patients undergoing open resection compared with those undergoing the VATS approach $(P=.042)$. Therefore, it appears that the LOS was not confounded by surgeon preference and was likely a true difference, reflecting the potentially shorter recovery time associated with a VATS approach.

As listed in Table 2, no significant differences were found in the co-morbidities between the 2 groups (median Charlson co-morbidity index score of 2 for the open group vs 3 for the VATS group, $P=.294$ ). The LOS was not associated with the Charlson co-morbidity index, as evaluated by testing the significance of the Spearman rank correlation coefficient (correlation $=.023, P=.884$ ).

\section{Comparison of Surgical Approaches by Propensity Score}

We compared the 2 surgical procedure groups with the aid of a propensity score. We evaluated the following candidates for their utility in the propensity score: Charlson co-morbidity index (raw and age adjusted), patient age and gender, tumor size and stage, date of surgery, and the presence of MG. Three covariates were selected because of their association with the surgical procedure groups: tumor size, date of surgery, and the presence of MG. No differences were found in overall survival, adjusting for the propensity score. In addition, no differences were found in recurrence-free survival, adjusting for the propensity score. The propensity score was independent of the LOS, and an analysis of covariance for the effect of surgical approach on the LOS remained significant $(P=.0043$, adjusting for propensity score).

\section{DISCUSSION}

Open surgical resection has been the standard treatment of patients with early-stage thymoma. In patients with MG, the approaches and extent of resection have been debated in published reports. These approaches have included transcervical thymectomy, trans-sternal thymectomy, video-assisted thoracoscopic thymectomy, and "maximal thymectomy," with separate sternal and cervical incisions. ${ }^{14,21-25}$ Minimally invasive approaches, including a transcervical approach and a VATS approach, have been described for thymoma; however, minimal data are available from published 
studies evaluating the outcomes to support the treatment of thymoma using these approaches.

In the present series, we analyzed 40 patients who had undergone resection for early-stage thymoma. Approximately one half of the patients had undergone total thymectomy using a VATS approach and one half had undergone resection with an open technique. This is the largest series of the published studies to our knowledge to compare these techniques for the treatment of thymoma. We found that minimally invasive VATS resection is safe and feasible. In addition, it was associated with a shorter hospital LOS. The oncologic outcomes were comparable between the open and VATS groups during intermediate-term followup. The present study primarily demonstrates the feasibility of a minimally invasive VATS approach for the resection of thymoma. It is important, however, to emphasize that thymoma is an indolent disease, and longer follow-up is required to evaluate and compare the oncologic outcomes.

\section{Minimally Invasive Approaches to Thymectomy}

Meyer and colleagues ${ }^{24}$ presented their results from a comparative study of trans-sternal versus minimally invasive thymectomy for patients with MG, primarily nonthymoma MG. A total of 48 patients underwent thymectomy with a minimally invasive approach and 47 patients with a trans-sternal approach. The series by Meyer and colleagues $^{24}$ included 10 patients with thymoma, 4 in the VATS group and 6 in the open group. Similar to the results from the present study, Meyer and colleagues ${ }^{24}$ noted a significant difference in the hospital LOS, favoring the minimally invasive group (1.9 vs 4 days). The 2 groups had equivalent improvement in the symptoms of MG. These investigators concluded that thymectomy was effective in MG, regardless of the surgical approach. Bachmann and colleagues ${ }^{25}$ compared the outcomes of thoracoscopic $(n=22)$ and open $(n=106)$ thymectomy in patients with MG. In their series, 27 patients had thymoma. Similar to our series, the hospital LOS was shorter with the thoracoscopic approach.

Transcervical thymectomy is another minimally invasive approach for the resection of the thymus. ${ }^{14,23}$ However, its use in patients with thymoma has also been controversial. One of the largest experiences was reported by Papatestas and colleagues ${ }^{26}$ of 46 patients with occult thymoma. More recently, Deeb and colleagues ${ }^{27}$ reported a series of 9 patients with thymoma who had undergone successful transcervical thymectomy. At a mean follow-up of 48 months, no recurrences had developed.

\section{Length of Stay}

In this study, we found that a minimally invasive VATS approach is associated with a shorter LOS in the hospital when compared with an open approach. There are, however, several confounding variables when analyzing the LOS in the hospital, and the LOS is not likely to be solely depen- dent on a single factor such as the surgical approach. These potential factors that can contribute to the LOS include selection bias, the experience and preference of the surgeon, patient co-morbidities, perioperative course, and social factors. Because our study was retrospective, the exact reason for the timing of discharge for the patients and the surgeon to surgeon variability in approving discharge was difficult to determine. In the present series, 5 surgeons performed most of the operations. Of the 5, 4 performed both VATS and open resection, and 1 performed open resection exclusively. When the results for the primary surgeons, who performed surgery using one or both of these approaches, were analyzed, open resections were associated with a longer LOS compared with VATS resections. Despite this finding, it is important to recognize that the underlying reasons for the hospital LOS are multifactorial and cannot be attributed to a single factor such as the surgical approach alone. Therefore, firm conclusions cannot be drawn regarding this issue owing to the inherent limitations of a retrospective study.

\section{Oncologic Outcomes}

A recent systematic review of surgical resection for thymoma reported that the overall 5-year survival rate of patients with stage I and stage II thymoma ranged from $89 \%$ to $100 \%$ and $71 \%$ to $95 \%$, respectively. ${ }^{10}$ That review also concluded that the published evidence comparing the outcomes of various surgical approaches, in particular, minimally invasive approaches, was insufficient. Although some reports have been published of thoracoscopic thymectomy for nonthymoma MG, very few data describing the outcomes for minimally invasive resection of thymoma are available. In one of the few studies exploring VATS approaches for thymoma, Cheng and associates ${ }^{28}$ compared VATS resection of stage II thymoma in 12 patients with open resection in 10 patients. They found no significant differences in the outcomes of either survival or recurrence during follow-up.

In addition to the tumor characteristics, ${ }^{29}$ one of the confounding variables in the evaluation of outcomes after thymectomy is the use of adjuvant therapy. Adjuvant radiotherapy is typically used for patients with stage II thymoma, although the value of adjuvant therapy has been questioned. ${ }^{30}$ In the present series, no significant differences were present in the use of adjuvant therapy between the groups undergoing minimally invasive versus open resection.

\section{Study Limitations}

The present study had several limitations. These limitations included those common to nonrandomized retrospective studies, such as the potential selection bias of the treating surgeon. In addition, the study sample was small, and few outcome events occurred. Although we did not find any significant difference between the 2 arms in terms 
of overall and recurrence-free survival, longer follow-up is required, because a full evaluation of the survival endpoints will require greater maturity of time-to-event data. Thymoma is an indolent disease, and longer follow-up is needed to determine the oncologic outcomes of VATS thymectomy. In addition, the use of the minimally invasive VATS approach was started later than use of the open procedure; thus, the VATS patients require additional observation for follow-up equivalence.

\section{CONCLUSIONS}

To our knowledge, the present series is the largest published report comparing the outcomes of a minimally invasive thoracoscopic resection of thymoma with those of an open technique. VATS resection of early-stage thymoma appears feasible and safe and was associated with a shorter hospital LOS. The oncologic outcomes also appear equivalent in the open and VATS groups during intermediate-term follow-up. Additional follow-up is required to evaluate the long-term results of thoracoscopic thymectomy for earlystage thymoma.

\section{References}

1. Lardinois D, Weder W. Diagnostic strategies in mediastinal mass. In: Patterson GA, Cooper JD, Deslauriers J, Lerut AEMR, Luketich JD, Rice TW, eds. Pearson's thoracic and esophageal surgery. 3rd ed. Philadelphia, Pa: Churchill Livingstone; 2008:1506-20.

2. Detterbeck FC, Parsons AM. Thymic tumors: A review of current diagnosis, classification and treatment. In: Patterson GA, Cooper JD, Deslauriers J, Lerut AEMR, Luketich JD, Rice TW, eds. Pearson's thoracic and esophageal surgery. 3rd ed. Philadelphia, Pa: Churchill Livingstone; 2008:1589-614.

3. Singhal S, Kaiser LR. Surgery for myasthenia gravis. In: Patterson GA, Cooper JD, Deslauriers J, Lerut AEMR, Luketich JD, Rice TW, eds. Pearson's thoracic and esophageal surgery. 3rd ed. Philadelphia, Pa: Churchill Livingstone; 2008:1549-61.

4. Schuchert MJ, Luketich JD, Landreneau RJ, Kilic A, Gooding WE, AlveloRivera M, et al. Minimally-invasive esophagomyotomy in 200 consecutive patients: Factors influencing postoperative outcomes. Ann Thorac Surg. 2008; 85:1729-34.

5. Pennathur A, Luketich JD, Heron DE, Abbas G, Burton S, Chen M, et al. Stereotactic radiosurgery for the treatment of stage I non-small cell lung cancer in highrisk patients. J Thorac Cardiovasc Surg. 2009;137:597-604.

6. Luketich JD, Alvelo-Rivera M, Buenaventura PO, Christie NA, McCaughan JS, Litle VR, et al. Minimally invasive esophagectomy: Outcomes in 222 patients. Ann Surg. 2003;238:486-95.

7. Onaitis MW, Petersen RP, Balderson SS, Toloza E, Burfeind WR, Harpole DH Jr, et al. Thoracoscopic lobectomy is a safe and versatile procedure: Experience with 500 consecutive patients. Ann Surg. 2006;244:420-5.

8. McKenna RJ, Houck W, Fuller CB. Video-assisted thoracic surgery lobectomy: Experience with 1100 cases. Ann Thorac Surg. 2006;81:421-6.

9. Blalock A, McGehee HA, Ford FR, et al. The treatment of myasthenia gravis by removal of the thymus gland. JAMA. 1941;117:1529-33.

10. Davenport E, Malthaner RA. The role of surgery in the management of thymoma: A systematic review. Ann Thorac Surg. 2008;86:673-84.

11. Mack MJ, Landreneau RJ, Yim AP, Hazelrigg SR, Scruggs G. Results of videoassisted thymectomy in patients with myasthenia gravis. $J$ Thorac Cardiovasc Surg. 1996;112:1352-60.

12. Landreneau RJ, Dowling RD, Castillo WM, Ferson PF. Thoracoscopic resection of an anterior mediastinal tumor. Ann Thorac Surg. 1992;54:142-4.

13. Yim AP. Video-assisted thoracoscopic resection of anterior mediastinal masses. Int Surg. 1996;81:350-3.

14. Cooper JD, Al-Jilaihawa AN, Pearson FG, Humphrey JG, Humphrey HE. An improved technique to facilitate transcervical thymectomy for myasthenia gravis. Ann Thorac Surg. 1988;45:242-7.
15. Trastek VF. Thymectomy. In: Kaiser LR, Kron IL, Spray TL, eds. Mastery of cardiothoracic surgery. Philadelphia, Pa: Lippincott William \& Wilkins; 1998. p. 105-11.

16. Charlson ME, Pompei P, Ales KL, MacKenzie CR. A new method of classifying prognostic comorbidity in longitudinal studies: development and validation. $J$ Chronic Dis. 1987;40:373-83.

17. Masaoka A, Monden Y, Nakahara K, Tanioka T. Follow-up study of thymomas with special reference to their clinical stages. Cancer. 1981;48:2485-92.

18. Osserman KE, Genkins G. Studies in myasthenia gravis: Review of a twenty-year experience in over 1200 patients. Mt Sinai J Med. 1971;38: 497-537.

19. Jaretzki A III, Barohn RJ, Ernstoff RM, Kaminski HJ, Keesey JC, Penn AS, et al., for the Task Force of the Medical Scientific Advisory Board of the Myasthenia Gravis Foundation of America. Myasthenia gravis: Recommendations for clinical research standards. Ann Thorac Surg. 2000;70:327-34.

20. Blackstone EH. Comparing apples and oranges. J Thorac Cardiovasc Surg. 2002; 123:8-15.

21. Detterbeck FC, Scott WW, Howard JF Jr, Egan TM, Keagy BA, Starek JK, et al. One hundred consecutive thymectomies for myasthenia gravis. Ann Thorac Surg. 1996;62:242-5.

22. Jaretzki A III, Penn AS, Younger DS, Wolff M, Olarte MR, Lovelace RE, et al. "Maximal" thymectomy for myasthenia gravis: Results. J Thorac Cardiovasc Surg. 1988;95:747-57.

23. Shrager JB, Nathan D, Brinster CJ, Yousuf O, Spence A, Chen Z, et al. Outcomes after 151 extended transcervical thymectomies for myasthenia gravis. Ann Thorac Surg. 2006;82:1863-9.

24. Meyer DM, Herbert MA, Sobhani NC, Tavakolian P, Duncan A, Bruns M, et al. Comparative clinical outcomes of thymectomy for myasthenia gravis performed by extended transsternal and minimally invasive approaches. Ann Thorac Surg. 2009;87:385-91.

25. Bachmann K, Burkhardt D, Schreiter I, Kaifi J, Busch C, Thayssen G, et al. Long-term outcome and quality of life after open and thoracoscopic thymectomy for myasthenia gravis: Analysis of 131 patients. Surg Endosc. 2008;22: 2470-7.

26. Papatestas AE, Pozner J, Genkins G, Kornfeld P, Matta RJ. Prognosis in occult thymomas in myasthenia gravis following transcervical thymectomy. Arch Surg. 1987;122:1352-6.

27. Deeb ME, Brinster CJ, Kucharzuk J, Shrager JB, Kaiser LR. Expanded indications for transcervical thymectomy in the management of anterior mediastinal masses. Ann Thorac Surg. 2001;72:208-11.

28. Cheng YJ, Kao EL, Chou SH. Videothoracoscopic resection of stage II thymoma: Prospective comparison of the results between thoracoscopy and open methods. Chest. 2005;128:3010-2.

29. Wright CD, Wain JC, Wong DR, Donahue DM, Gaissert HA, Grillo HC, et al. Predictors of recurrence in thymic tumors: Importance of invasion, World Health Organization histology, and size. J Thorac Cardiovasc Surg. 2005;130: 1413-20.

30. Mangi AA, Wright CD, Allan JS, Wain JC, Donahue DM, Grillo HC, et al. Adjuvant radiation therapy for stage II thymoma. Ann Thorac Surg. 2002;74:1033-7.

\section{Discussion}

Dr Nasser Altorki (New York, NY). I have a question for you. I disagree with 4 of your 5 conclusions. I think to talk about oncologic validity in thymoma with anything that is less than 5 years, or preferably 10 years, is probably not correct. Thirty six months is just way too short of a follow-up to be able to tell that. How did you follow-up those patients? How carefully did you followup those patients?

Dr Pennathur. Well, we have acknowledged in our conclusions that longer follow-up is required. We saw all of these patients in the clinic typically about every 4 to 6 months. Now, the myasthenia patients were also followed very closely by the neurologist, and that follow-up was actually a little bit closer, about 3 months. So I would say that the myasthenia patients were a little bit more closely followed-up compared with the nonmyasthenic thymoma patients.

Dr Altorki. My concern is that I have seen some of those patients come after transpleural resections, and sometimes you 
have to enter the pleural space and they will come back 10 years later, 12 years later, with pleural implants in the posterior gutters, basically in both pleural spaces, and that is one reason that I have found the video-assisted thoracoscopic surgery approach a little bit intimidating, at least for me.

Dr Pennathur. Yes, I think your point is very valid. I agree that we need longer follow-up, to fully evaluate the results, which we have acknowledged in the conclusions. However, there is no series in the literature to my knowledge evaluating outcomes comparing a thoracoscopic approach to an open approach at this length of follow-up (36 months), and that is what we have attempted to do. Also, in terms of the pleural droplet metastasis which does occur in thymoma, the thoracoscopic approach provides good visualization. The first thing you do is explore the chest and we are able to actually get a good view of the pleura space with the thoracoscopic approach.

Dr Federico Venuta (Rome, Italy). I was reading your abstract and I noted in the abstract that you reported 3 patients with positive margins but in your presentation there was only 1 patient with a positive margin in the thoracoscopic group.

Dr Pennathur. Yes. That is an error in the abstract. It is 1 patient with a positive margin.

Dr Venuta. And if I may ask another question, how did you divide these patients with the 2 approaches? How did you decide who received the open approach and who received the thoracoscopic approach?

Dr Pennathur. This is certainly not a randomized study, so there is clearly a selection bias in terms of the surgeon and the patient in deciding the approach. In general, if the thymoma is less than about $5 \mathrm{~cm}$ or so, particularly in the early stage of performing these thoracoscopic thymectomies, we preferred to do those thymomas that looked small and encapsulated. Some of them on final pathology, however, had capsular invasion, putting them as a stage II. When there was concern about invasion into adjacent structures or bigger lesions, those were handled by an open approach. The largest lesion in this series in the video-assisted thoracoscopic surgery approach was $5.6 \mathrm{~cm}$ and the median was $3.6 \mathrm{~cm}$. So I think it is the size, the appearance on the CT scan, relationship to adjacent structures, and, of course, there is a selection bias in terms of the surgeon who is doing the case. This is a retrospective study. It's not a prospective randomized study.

Dr Venuta. What are your indications for postoperative radiotherapy?

Dr Pennathur. Well, I know this is being debated. Dr Wright at Massachusetts General had presented a series on the usefulness of radiation for stage II and a similar series has been presented by Dr Singhal from the University of Pennsylvania questioning the value of radiation. In our institution, if it's a stage II or higher, we give consideration for radiation therapy. Other considerations for radiation include positive margins and more advanced tumors. But I think this is something is being questioned. The recurrences occur many times in the pleura, as Dr Altorki pointed out, so radiating them in the chest - the thymic bed-may not entirely be effective.
Dr Raphael Bueno (Boston, Mass). Nice paper and I think it will take time to figure out what the answer is. You had another parameter besides oncologic, which is the recurrence of myasthenia. Did that differ in the 2 groups?

Dr Pennathur. No, it did not differ. The bottom line was that the Osserman score showed a significant improvement in both the groups. Nine out of 11 patients with myasthenia improved. The Osserman score had a mean drop of negative 2, which is an overall improvement. We did not find significant differences between the surgical groups.

Dr Michael Jaklitsch (Boston, Mass). My personal inhibition against doing a thoracoscopic thymectomy for thymoma is from my personal experience, in that in dissecting out the gland, I can see micro-defects in the capsule of the gland, and what makes you stage I is having that gland intact. I have used a variety of techniques, but I can see these little nicks, which I do not get with a partial sternotomy. Am I different from you and you have a technique that you can get the gland out without capsular nicks, or am I like you and we are both getting capsular nicks but you are showing me oncologically that those nicks do not matter?

Dr Pennathur. Well, I think that there are various ways of mobilizing the thymus, and one way, for example, described by $\mathrm{Dr} \mathrm{Au}-$ gasthian at the Society of Thoracic Surgeons, which is similar to ours, is we do not handle the tumor first. Try to go toward more of the normal thymus, mobilize, and create the plane before obviously going where the mass is and before trying to mobilize the mass initially. So, if you start from the normal tissue and start mobilizing from there, that helps. The other thing that we have done over the years with the evolution of the technique is that we have used a bilateral approach. You get a very good look at the left side. We start mobilization on the left side, and then as we move along, the patient is placed supine, which also helps with easy conversion, if needed; and then we go to the right side, and, again, you can take from the normal tissue and then mobilize it, and then you will be able to potentially avoid some of those nicks. But it can be a problem. One has to handle the capsule very carefully.

Dr Thomas Rice (Cleveland, Ohio). Beyond the short followup and the few events, you have to ask: Are these comparable groups? So when you come to doing your multivariable analysis when you get enough events, even if you do not have enough patients, you should put the propensity score in the multivariable analysis to see if it is a fair comparison. It probably is not.

Dr Pennathur. Dr Rice, thank you for your comments. Yes, we can certainly consider doing that-trying to adjust for the various variables. One thing we found was that size was bigger in the open group.

Dr Nasser Altorki (New York, NY). Perhaps you have done it already, but let me just make a pitch for the partial sternal splits, which are ideally suited for small thymomas of the superior mediastinum, because I do agree that your length of stay can probably be shortened.

Dr Pennathur. Yes. Thanks for your comments. I want to thank the Society for the opportunity to present this paper. 